\title{
Enhanced bone regeneration using an insulin- loaded nano-hydroxyapatite/collagen/PLGA composite scaffold
}

This article was published in the following Dove Press journal: International Journal of Nanomedicine

\author{
Xing Wang ${ }^{1, *}$ \\ Guilan Zhang ${ }^{2, *}$ \\ Feng $\mathrm{Qi}^{3}$ \\ Yongfeng Cheng' \\ Xuguang Lu' \\ Lu Wang' \\ Jing Zhao' \\ Bin Zhao'
}

'Shanxi Medical University Stomatological Hospital, Taiyuan, ${ }^{2}$ Department of Stomatology, Nanfang Hospital, Southern Medical University, Guangzhou, China; ${ }^{3}$ Department of Mechanical and Aerospace Engineering, University of Missouri, Columbia, MO, USA

*These authors contributed equally to this work
Correspondence: Bin Zhao

Shanxi Medical University Stomatological Hospital, Xinjian South Road 63\#,

Taiyuan 03000I, China

$\mathrm{Tel} / \mathrm{fax}+8635 \mathrm{I} 4690307$

Email sxkqyy@I63.com

\begin{abstract}
Insulin is widely considered as a classical hormone and drug in maintaining energy and glucose homeostasis. Recently, insulin has been increasingly recognized as an indispensable factor for osteogenesis and bone turnover, but its applications in bone regeneration have been restricted because of the short periods of activity and uncontrolled release. In this study, we incorporated insulin-loaded poly lactic-co-glycolic-acid (PLGA) nanospheres into nanohydroxyapatite/collagen (nHAC) scaffolds and investigated the bioactivity of the composite scaffolds in vitro and in vivo. Bioactive insulin was successfully released from the nanospheres within the scaffold, and the release kinetics of insulin could be efficiently controlled by uniformsized nanospheres. The physical characterizations of the composite scaffolds demonstrated that incorporation of nanospheres in nHAC scaffolds using this method did not significantly change the porosity, pore diameters, and compressive strengths of nHAC. In vitro, the insulin-loaded nHAC/PLGA composite scaffolds possessed favorable biological function for bone marrow mesenchymal stem cells adhesion and proliferation, as well as the differentiation into osteoblasts. In vivo, the optimized bone regenerative capability of this composite scaffold was confirmed in rabbit mandible critical size defects. These results demonstrated successful development of a functional insulin-PLGA-nHAC composite scaffold that enhances the bone regeneration capability of nHAC.
\end{abstract}

Keywords: insulin, composite scaffold, drug delivery system, bone tissue engineering

\section{Introduction}

A wide variety of biomaterials have been employed in bone regeneration. Generally, research on the development of new biomaterials mainly focuses on the fabrication of three-dimensional micro/nanoscale structures or functional scaffolds capable of adhering osteoblasts. Recently, there is a growing interest in the development of porous scaffolds for delivery of drugs or growth factors. These scaffolds can regulate specific functions of bone marrow mesenchymal stem cells (BMSCs), including gene expression processes associated with cell growth and osteogenic differentiation. ${ }^{1,2}$

Insulin has been increasingly accepted as an inducible factor for bone turnover and osteogenesis. BMSCs have been proved to express functional insulin receptors and respond to exogenous insulin by increasing proliferation rates, ${ }^{3}$ alkaline phosphatase (ALP) production, ${ }^{4}$ and collagen synthesis, ${ }^{5}$ which is associated with the upregulation of PI3-K/Akt in cells. ${ }^{6}$ Our previous study demonstrated that insulin induced bone formation improvement by twice or thrice the initial level when administered locally. ${ }^{7}$ Although there have been evidences to suggest that insulin induces osteogenesis, little attention has been given to its application in bone regeneration. However, 
as the half-life of insulin in plasma is only 5-15 minutes, the applications of insulin in vivo are limited by short periods of insulin activity, as well as uncontrolled release. Therefore, a controlled, localized insulin delivery system to maintain bioactivity and drug concentration at the defect site for effective bone regeneration is required.

Poly lactic-co-glycolic-acid (PLGA) possesses excellent biocompatibility and biodegradability, and has been extensively studied for drug delivery. ${ }^{8}$ In terms of bone tissue engineering, the ideal material would serve not only as a drug delivery carrier, but also as a porous scaffold for cellular activities. ${ }^{9}$ Recently, nano-hydroxyapatite/collagen (nHAC) scaffolds have been proved to be promising for cell adhesion and proliferation. ${ }^{10}$ However, it is still difficult to regulate drug release behavior before $\mathrm{nHAC}$ degradation.

Therefore, a composite PLGA and nHAC scaffold may provide an alternative, and can be used as a three-dimensional porous scaffold for cell growth and insulin delivery. Based on this, we incorporated insulin-loaded PLGA nanospheres into porous nHAC scaffolds, tested the biological activity of the composite scaffolds, and investigated the bone regeneration potential of the composite scaffolds in a critical size defect model.

\section{Materials and methods Materials}

The molar ratio of PLGA used was D,L-lactide/glycolide 75/25 (molecular weight $13 \mathrm{kDa}$ ) (Lakeshore Biomaterials, Birmingham, AL, USA). The nHAC scaffolds were characterized as follows: hydroxyapatite content of $45 \% \pm 5 \%$, porosity of $75 \%-88 \%$, and pore size of $80-150 \mathrm{~nm} \cdot{ }^{11,12}$ Human recombinant insulin was obtained from Wako Pure Chemical Industries, Ltd (Osaka, Japan). nHAC was purchased from Beijing Allgens Medical Science \& Technology Co, Ltd (Beijing, China). Sodium cholate was purchased from Sigma-Aldrich Co (St Louis, MO, USA). All other reagents were of analytical grade.

\section{Preparation of insulin-loaded nanospheres}

Nanospheres were prepared by W1/O/W2 double emulsion solvent evaporation method. ${ }^{13}$ One milliliter of insulin solution $(3 \%, \mathrm{w} / \mathrm{v}, \mathrm{W} 1)$ and $8 \mathrm{~mL}$ of methylene dichloride containing PLGA $(10 \%, \mathrm{w} / \mathrm{v})$ were ultrasonicated at $200 \mathrm{~W}$ for 50 seconds forming primary emulsions (W1/O). The coarse double emulsions (W1/O/W2) were formed by mixing the $\mathrm{W} 1 / \mathrm{O}$ emulsions with an external aqueous phase containing sodium cholate hydrate $(1 \%, \mathrm{w} / \mathrm{v})$. Next, the W1/O/W2 emulsions were stirred at $600 \mathrm{rpm}$ for 5 minutes and were solidified at $250 \mathrm{rpm}$ for 5 hours at room temperature. Finally, the nanospheres were washed with a 100,000 Da ultrafiltration membrane 5 times, and collected. Blank PLGA nanospheres were fabricated using the same method, but distilled water was used as the internal aqueous phase.

\section{Surface morphology observation and size distribution measurement}

The surface morphology and shape of PLGA nanospheres were observed by a scanning electron microscope (SEM; JSM-6700 F; JEOL, Tokyo, Japan). Particle size distribution and size were measured by laser diffraction using Zetasizer Nano (Malvern Instruments, Malvern, UK). The size uniformity of particles was referred as polydispersity index (PDI).

\section{Measurement of insulin loading efficiency (LE) and encapsulation efficiency (EE)}

PLGA nanospheres $(5 \mathrm{mg})$ were resuspended in $\mathrm{NaOH}$ solution $(0.04 \mathrm{M}, 10 \mathrm{~mL})$ on an orbital shaker at constant $110 \mathrm{rpm}$ gentle shaking at $37^{\circ} \mathrm{C}$ for 12 hours. A micro bicinchoninic acid (BCA) protein assay kit (Fanbo Biochemicals, Beijing, China) was used for quantitative measurement of insulin loaded in the PLGA nanospheres. Blank PLGA nanospheres were used as control. LE and EE of the particles were calculated using the following equations:

$$
\begin{aligned}
& \operatorname{LE}(\%, w / w)=\frac{\text { Mass of drug in nanospheres }}{\text { Mass of nanospheres }} \times 100 \% \\
& \operatorname{EE}(\%, w / w)=\frac{\text { Loading efficiency }}{\text { Theoretical loading efficiency }} \times 100 \%
\end{aligned}
$$

\section{Drug distribution in the PLGA nanospheres}

To observe drug distribution in the PLGA nanospheres, insulin labeled with Super Fluor 488 SE (Fanbo Biochemicals) was encapsulated into PLGA nanospheres. Confocal laser scanning microscopy (CLSM; Leica Microsystems, Wetzlar, Germany) was used to observe PLGA nanospheres at $488 \mathrm{~nm}$ excitation wavelength.

\section{Insulin release from the PLGA nanospheres}

Insulin-loaded PLGA nanospheres $(20 \mathrm{mg})$ were placed in a 10,000 Da bag filter and resuspended in $10 \mathrm{~mL}$ of $10 \mathrm{mM}$ phosphate-buffered saline medium ( $\mathrm{pH} 7.4$ ). The suspension was stirred at $30 \mathrm{rpm}$ and at $37^{\circ} \mathrm{C}$. At $6,12,24,36$ hours, 
and 2-6 days, the medium was taken from the bag filter and replaced with an equal volume of fresh buffer. The concentrations of insulin in the collected medium were measured using an insulin enzyme-linked immunosorbent assay (ELISA) Kit (Mercodia, Uppsala, Sweden). 100 mU/L insulin solution was used as a control. Measurements were carried out three times.

\section{Fabrication of nHAC/PLGA composite scaffolds}

Firstly, an insulin-loaded PLGA nanospheres suspension $(0.5 \%, w / v, 4 \mathrm{~mL})$ was prepared. A $20 \mathrm{mg}$ mass of the nHAC scaffold was immersed into the PLGA nanospheres suspension under $0.1 \mathrm{MPa}$ vacuum suction for 10 minutes and subsequently vortexed for 10 minutes (I-nHAC/PLGA). As a control, $20 \mathrm{mg}$ of the nHAC scaffold was immersed into blank PLGA nanospheres suspensions (nHAC/PLGA), and the same procedure was repeated. nHAC scaffold sample (20 mg) immersed into a $2 \mathrm{~mL}$ insulin solution $(0.5 \%, \mathrm{w} / \mathrm{v})$ under $0.1 \mathrm{MPa}$ vacuum suction was employed as another control group (I-nHAC). The prepared suspensions were centrifuged, and the unloaded PLGA nanospheres were collected after vacuum suctioning. The remaining insulin in solution was measured using micro BCA assay kits. The amount of insulin loaded into the nHAC scaffolds was determined by subtracting the remaining insulin from the initial amount. ${ }^{14}$ Measurements were carried out three times.

\section{Physical characterizations of scaffolds}

The effects of the added nanospheres on the mechanical behavior of scaffolds were evaluated and compared with unloaded nHAC scaffolds. Morphological characteristics of the surfaces and cross-sections obtained from the nHAC/ PLGA composite scaffolds were observed using SEM. To quantitatively analyze the nHAC/PLGA composite support structure, the scaffold was embedded in glycol methacrylate (Polysciences, Warrington, PA, USA), sectioned, and stained with toluidine blue. To calculate pore diameters and porosity of the scaffolds, the stained images were processed and subjected to uneven lighting removal, image enhancement, binarization, and interference objectives removal, using pore topology analyzer software (MATLAB, Natick, MA, USA). ${ }^{15}$ Using a universal testing machine (Z050; Zwick/ Roell, Ulm, Germany) at a constant loading rate of $0.5 \mathrm{~mm} /$ min, the compressive strengths of the scaffold specimens (size of $10 \times 5 \times 3 \mathrm{~mm}^{3}$ ) were measured. ${ }^{16}$ The maximum point of the stress-strain curve was determined by the compressive strength. Measurements were carried out three times.

\section{Insulin release kinetics of scaffolds}

To examine the insulin release profiles, scaffolds $\left(10 \times 5 \times 3 \mathrm{~mm}^{3}\right.$ size) were placed in a 10,000 Da bag filter and incubated in $10 \mathrm{~mL}$ of $10 \mathrm{mM}$ phosphate-buffered saline medium (pH 7.4) in a shaking water bath at $37^{\circ} \mathrm{C}$. At $6,12,24,36$ hours, and 2-6 days, samples were collected, and the medium was replaced with an equal volume of fresh buffer. Insulin concentrations in the collected samples were measured using an insulin ELISA kit (Mercodia). Measurements were carried out three times.

\section{In vitro bioactivity assessment of insulin delivered from scaffolds}

Human BMSCs were isolated from bone marrow and cultured as described previously. ${ }^{17}$ Bone marrow specimens were obtained from femoral tissues discarded during primary hip arthroplasty with the approval of the Chinese PLA General Hospital Review Board. All the patients whose tissues were used in this research provided written informed consent. To minimize the potential external effects on BMSCs, the following exclusion criteria were used: rheumatoid arthritis, cancer, osteoporosis, diabetes, and ages $>50$ or $<30$. A total of 16 subjects, 8 women and 8 men, with ages from 34 to 49 years, were enrolled in this study. Not all specimens could be included in every experiment because of the surgical schedule and the number of cells needed for each assay. In each experiment, cells obtained from different subjects were stored for analysis at the same time to avoid technical differences between assays.

Briefly, $100 \mu \mathrm{L}$ of P4 BMSCs $\left(10^{6} / \mathrm{mL}\right)$ was dropwise seeded into nHAC, I-nHAC, nHAC/PLGA, and I-nHAC/ PLGA scaffold blocks $\left(10 \times 5 \times 3 \mathrm{~mm}^{3}\right)$ in individual wells of a 24-well plate. Minimum essential alpha medium ( $\alpha$ MEM; Thermo Fisher Scientific, Waltham, MA, USA) containing $15 \%$ fetal bovine serum (FBS, Thermo Fisher Scientific) was added to each well. The medium was renewed twice weekly. After 3, 7, and 14 days of culture, DNA assays were used to analyze the influence of the scaffolds on cell proliferation. Using a rotor-stator homogenizer (Omni International, Kennesaw, GA, USA), the scaffolds were homogenized in lysis buffer (SensoLyte pNPP Alkaline Phosphatase Assay Kit, AnaSpec, Fremont, CA, USA). Then, using Quanti-iT ${ }^{\mathrm{TM}}$ PicoGreen dsDNA Molecular Probes (Thermo Fisher Scientific), the resultant suspensions were centrifuged at $2,500 \times g$ for 10 minutes at $4{ }^{\circ} \mathrm{C}$ and analyzed for dsDNA content. ${ }^{18} \mathrm{~A}$ fixed number of cells was analyzed to convert the DNA content to the number of cells per sample. 
ALP and osteocalcin (OCN) activity levels were determined to evaluate the osteogenic differentiation of the BMSCs cultured in the scaffolds. After 3, 7, and 14 days, the supernatant was collected and replaced with equal amounts of fresh $\alpha M E M$ (with 15\% FBS). Using Roche Diagnostics ALP kits on a Cobas e602 platform (Hoffman-La Roche Ltd, Basel, Switzerland), the ALP activity in the medium was detected. OCN activity was measured by electrochemiluminescence immunoassay techniques using N-MID Osteocalcin kits (Hoffman-La Roche Ltd, Basel, Switzerland). The measurements were carried out three times, and all data were analyzed and measured on a Cobas 8000 platform (Hoffman-La Roche Ltd).

Similarly, $1 \times 10^{5}$ BMSCs were cultured on scaffolds in $\alpha \mathrm{MEM}$ (with 15\% FBS). At day 14, the cells were fixed with $2.5 \%$ glutaraldehyde and stained with Alizarin Red. The depositions were extracted using $10 \%(\mathrm{w} / \mathrm{v})$ cetylpyridinium chloride in $10 \mathrm{mM}$ sodium phosphate ( $\mathrm{pH} 7.0)$ to quantify the amount of Alizarin Red at room temperature for 2 hours. The amount of Alizarin Red in the extraction buffer was measured by the optical density (OD) of the solution at $560 \mathrm{~nm} .^{19}$

\section{In vivo studies of the restoration of critical size bone defects}

The bone regeneration ability of the composite scaffolds was estimated using a critical size mandible bone defect model of New Zealand white rabbits. All surgical procedures and care were conducted in accordance with the Animal Handling Guideline of Chinese PLA General Hospital and all animal procedures were approved by the Institutional Animal Care and Use Committee of Chinese PLA General Hospital. Forty female rabbits weighing between 2.50 and $3.00 \mathrm{~kg}$ were housed. The rabbits were anesthetized by intravenous injections of $2 \%$ sodium pentobarbital $(30 \mathrm{mg} / \mathrm{kg})$. In a sterile environment, a $15 \mathrm{~mm}$ incision was made from the edge of the right mandible of each rabbit. Segmental defects $\left(10 \times 5 \times 3 \mathrm{~mm}^{3}\right)$ were prepared in the mandible using a surgical oscillating saw, supplemented with sterile saline flush. ${ }^{20}$ Ten defects were treated with I-nHAC/PLGA composite scaffolds. Defects treated with nHAC, I-nHAC, and nHAC/ PLGA scaffolds were used as controls.

At 4 and 8 weeks after the surgical procedure, rabbits were sacrificed successively to collect mandible specimens. Then, the specimens were surgically removed, fixed, dehydrated, and embedded undecalcified in methyl methacrylate. Tissue sections (20 $\mu \mathrm{m}$ thick) were cut, ground, and stained with toluidine blue and Goldner's trichrome. All samples were observed under a Leica DMLB microscope and analyzed by BioQuant software (R\&M Biometrics, Nashville,
TN, USA). Ten successive histological sections were prepared from each implant according to Cavalierie's principle. ${ }^{21}$ The amount of bone formed was determined in each section as a percentage of bone volume per total volume (BV/TV) by point counting using a grid.22

\section{Statistical analysis}

All data are presented as the mean \pm SD. Statistical analyses of the results were performed using SPSS 16.0 software (SPSS Inc, Chicago, IL, USA). One-way analysis of variance and two-tailed Student's $t$-tests were used to determine significance. The significance threshold was set at $p<0.05$.

\section{Results \\ Characteristics of the insulin-loaded PLGA nanospheres}

Uniform-sized, insulin-loaded PLGA nanospheres with a narrow size distribution were successfully fabricated. The surface morphologies of the PLGA nanospheres were spherical and smooth, as shown in the SEM image (Figure 1A). Labeled insulin was well-distributed in the uniform-sized PLGA nanospheres, as shown in the CLSM image (Figure 1B). The particle size of the PLGA nanospheres was $83.73 \pm 2 \mathrm{~nm}$ $(\mathrm{PDI}=0.096, \mathrm{LE}=5.3 \% \pm 0.21 \%$, and $\mathrm{EE}=55.2 \% \pm 2.13 \%)$ (Figure 1C). The nanospheres exhibited an initial burst release ( $\sim 55 \%$ within 24 hours), followed by a sustained release of the remaining insulin over 3 days (Figure 1D).

\section{Physical characterizations of nHAC/PLGA composite scaffolds}

Figure $2 \mathrm{~A}$ and $\mathrm{B}$ shows that $\mathrm{nHAC}$ scaffolds exhibit a regular, porous hierarchical microstructure (with an average pore size of $\sim 120 \pm 20 \mu \mathrm{m})$. After PLGA fixation, the SEM images show that the nHAC/PLGA composite scaffolds maintain an interconnected pore structure, and the PLGA nanospheres maintain a smooth spherical shape. Most nanospheres successfully adhere to the scaffold sidewall and fix to the surface or small grooves of the nHAC (Figure 2C).

The pore diameters of the nHAC, I-nHAC, and I-nHAC/ PLGA composite scaffolds were 123.8 $\pm 20.6,116.4 \pm 23.2$, and 127.2 $\pm 24.3 \mu \mathrm{m}$, respectively (Figure $2 \mathrm{D}$ ). The porosities of the nHAC, I-nHAC, and I-nHAC/PLGA composite scaffolds were $80.6 \% \pm 2.7 \%, 79.3 \% \pm 2.5 \%$, and $79.8 \% \pm 2.6 \%$, respectively (Figure $2 \mathrm{E}$ ). The results of the quantitative analysis were in agreement with the results of SEM observations. The results also showed that the method used in the nHAC scaffolds preparation did not significantly change the porosity. Mechanical properties of I-nHAC/PLGA composite 
A
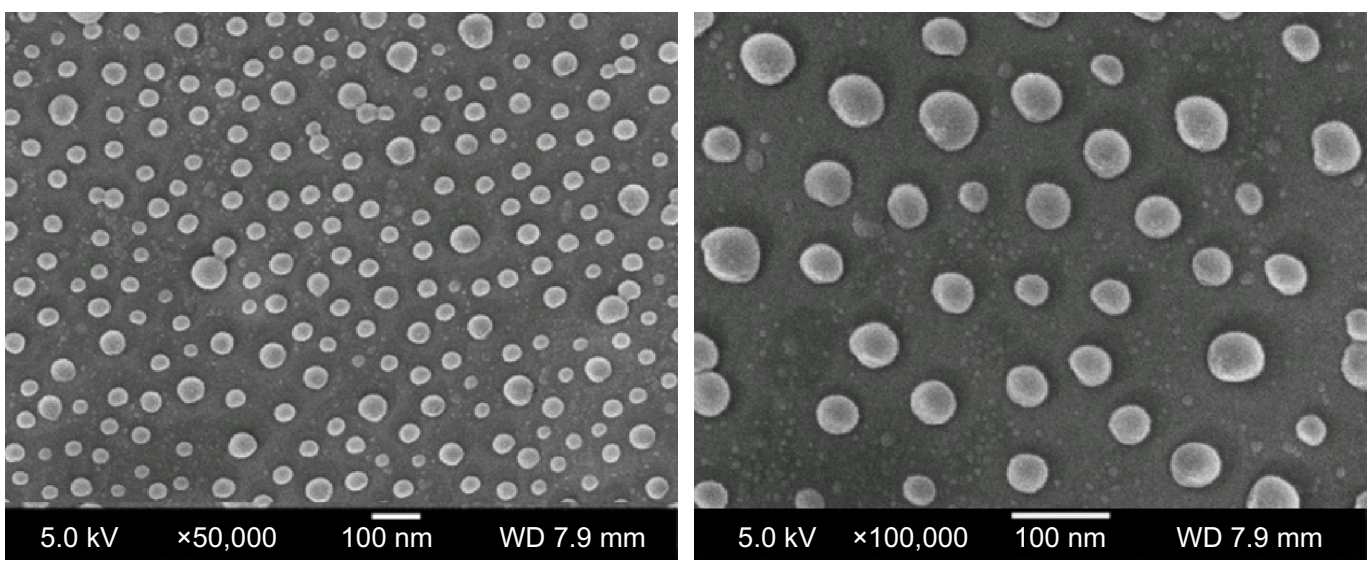

B
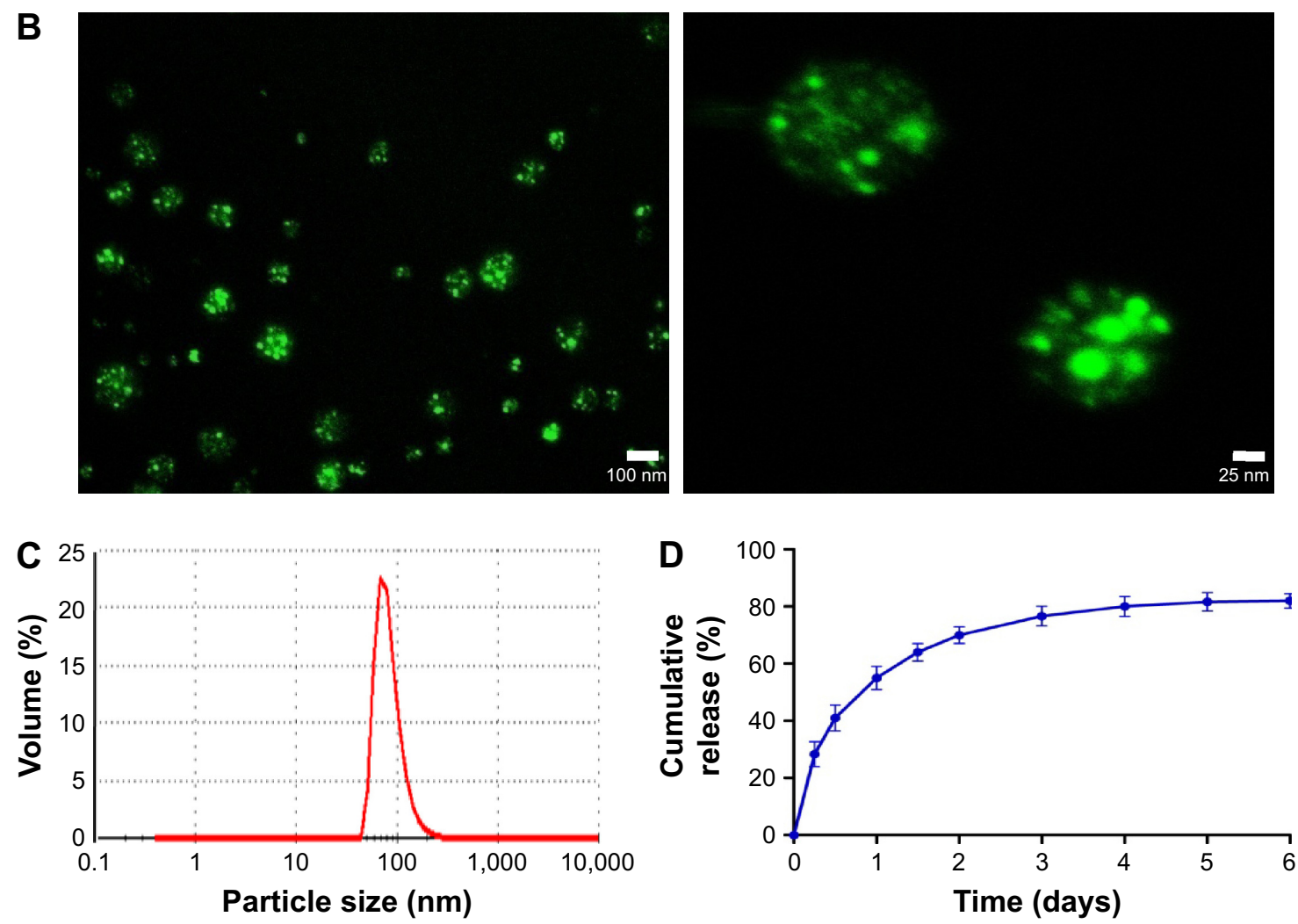

Figure I SEM images (A), CLSM images (B), size distribution (C), and in vitro release profiles (D) of insulin-loaded PLGA nanospheres.

Abbreviations: CLSM, confocal laser scanning microscopy; PLGA, poly lactic-co-glycolic-acid; SEM, scanning electron microscope.

scaffolds were evaluated using compressive measurements. As shown in Figure 2F, the incorporation of nanospheres in the nHAC had no significant effects on the compressive strengths of the scaffolds.

\section{Kinetics of insulin release from $\mathrm{nHACl}$ PLGA scaffolds}

As shown in Figure 3A, the insulin loading amount of the I-nHAC and I-nHAC/PLGA scaffolds was $8.7 \pm 1.4$ and $135.0 \pm 7.6 \mu \mathrm{g}$, respectively. The insulin load efficiency of the I-nHAC and I-nHAC/PLGA scaffolds were $0.08 \%$ and $12.7 \%$, respectively. I-nHAC scaffolds were loaded with a small amount of insulin and exhibited a rapid release rate $(93 \%$ within 24 hours). The I-nHAC/PLGA scaffolds exhibited an initial burst release ( $46 \%$ within 24 hours), followed by a constant release over 5 days. These results indicated that the I-nHAC/ PLGA scaffolds could continuously release insulin.

\section{In vitro cytocompatibility and bioactivity of scaffolds}

To investigate the effects of the scaffolds on cell proliferation, human BMSCs were cultured on the scaffolds, and 

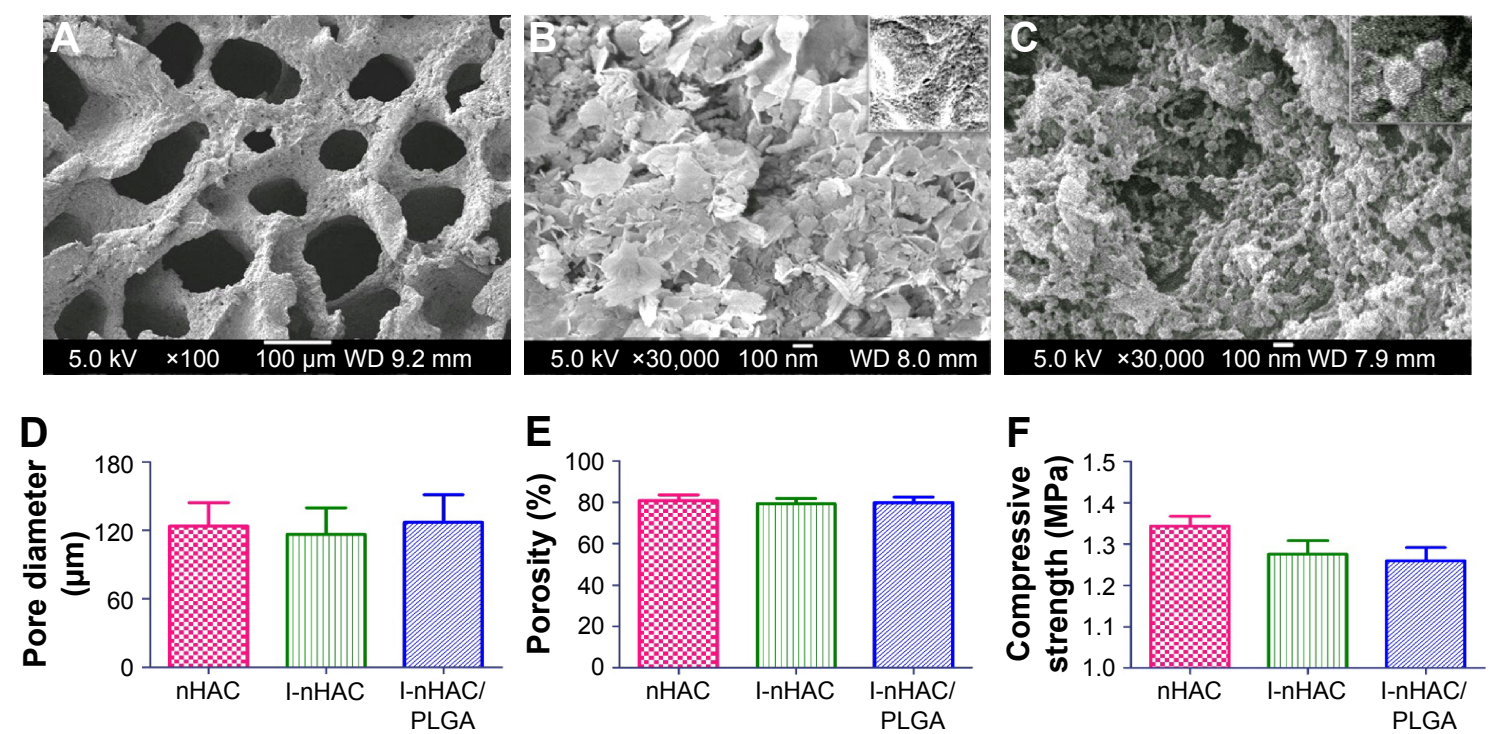

Figure 2 SEM images of the structure of the $\mathrm{nHAC}$ scaffold $(\mathbf{A}, \mathbf{B})$ and $\mathrm{nHAC/PLGA}$ composite scaffolds (C). Pore diameters (D), porosity (E), and compressive strengths (F) of the composite scaffolds.

Abbreviations: nHAC, nano-hydroxyapatite/collagen; PLGA, poly lactic-co-glycolic-acid; SEM, scanning electron microscope.

cell viability was evaluated. The cell number increased in all scaffolds throughout 14 days of culture (Figure 3B). Comparison of cells cultured on the nHAC, I-nHAC, and nHAC/PLGA scaffolds indicated that there was a significant
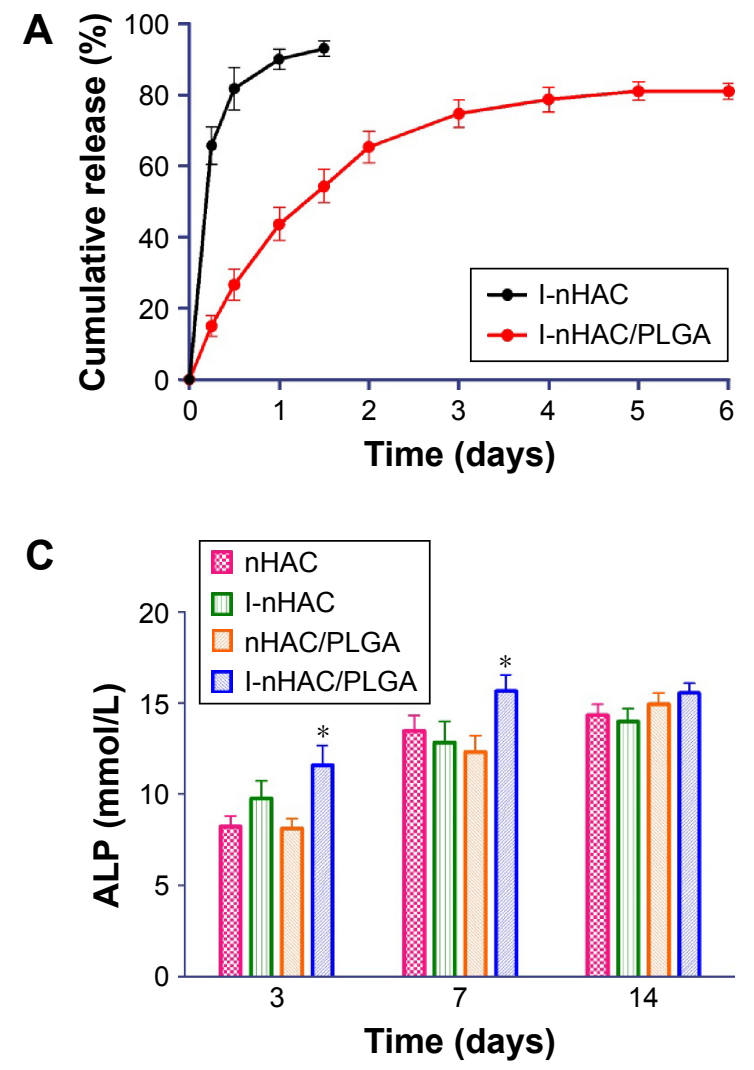

increase in the number of cells on the I-nHAC/PLGA scaffolds after 7 days.

BMSC differentiation along osteogenic lineages on the composite scaffolds was assessed by ALP and OCN assays
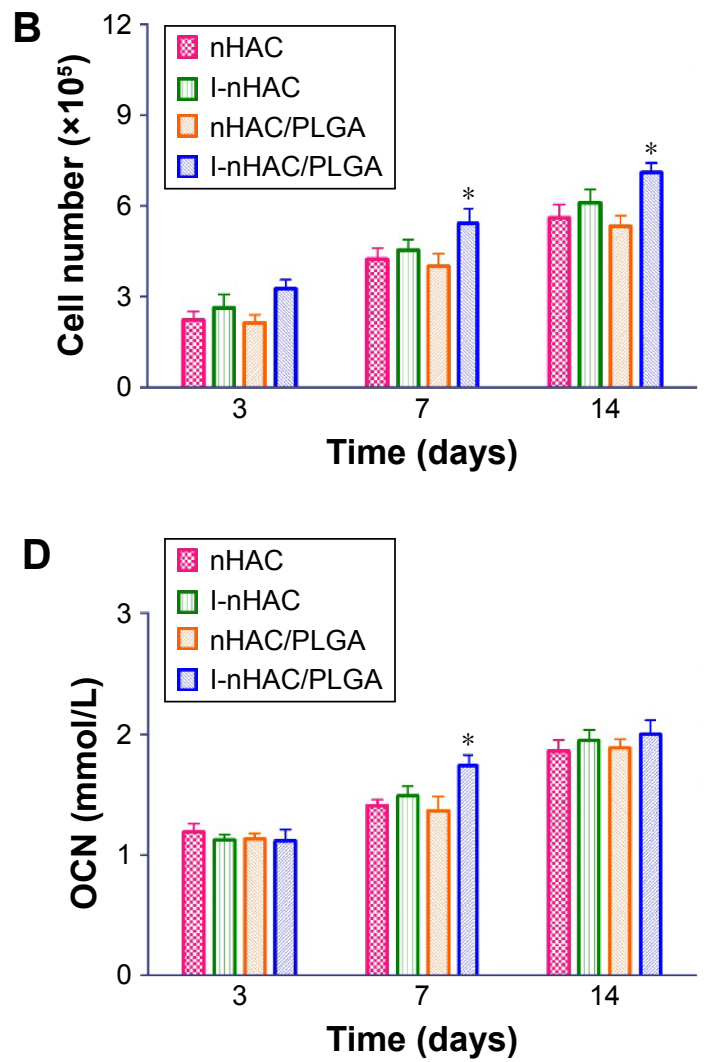

Figure 3 (A) In vitro release profiles of the I-nHAC and I-nHAC/PLGA scaffolds ( $n=3$, mean \pm SD). The number of BMSCs (B), ALP (C), and OCN concentration (D) in different groups. *Compared to $\mathrm{nHAC}$. Differences are significant at $p<0.05$.

Abbreviations: ALP, alkaline phosphatase; BMSCs, bone marrow mesenchymal stem cells; nHAC, nano-hydroxyapatite/collagen; OCN, osteocalcin; PLGA, poly lactic-coglycolic-acid. 
and Alizarin Red staining. At day 3 and 7, ALP concentrations in the I-nHAC/PLGA group were significantly higher than those of the other groups (Figure 3C). At day 14, there were no significant differences in the ALP concentrations among the different groups. At day 7, OCN concentration in the I-nHAC/PLGA group was significantly higher than those of the other groups (Figure 3D). At day 3 and 14, there were no significant differences in OCN concentrations among the different groups.

At day 14, mineralized matrix formations in vitro were evaluated by Alizarin Red staining. As shown in Figure 4, the color intensity of the I-nHAC/PLGA group was obviously higher than the other groups. Calcium accumulation was quantified to determine the extent of osteogenic differentiation. The OD ratios in the four groups were distinguishable (nHAC: $3.00 \pm 0.52$, I-nHAC: $3.23 \pm 0.43$, nHAC/PLGA: $3.07 \pm 0.44$, and I-nHAC/PLGA: 4.8 \pm 0.52 ). However, only the OD of I-nHAC/PLGA group was significantly higher than those of the other groups.

\section{In vivo evaluation of $n$ HAC/PLGA scaffolds}

Histological staining of mandible specimens containing the porous scaffolds after 4 and 8 weeks post-operation was conducted to determine tissue quality and bone formation. Toluidine blue staining is shown at different magnifications in Figure 5. In the fourth week, many regions had no observable bone formation in the nHAC, I-nHAC, and nHAC/PLGA groups. In contrast, the pore channels of the I-nHAC/PLGA scaffolds were filled with bone matrix in the defect area. High power microscopy showed that osteoblasts lined around the newly formed bone. In the eighth week, mature bone tissues were observed in the I-nHAC/PLGA group. High power microscopy showed that large numbers of osteocytes were located in the bone lacuna. In the nHAC, I-nHAC, and nHAC/ PLGA groups, a similar regeneration process was observed, but with a number of dark blue-stained osteoids and a few light blue-stained mature bones.

Goldner's trichrome staining also indicated a similar trend in Figure 6, wherein scaffolds (gray staining), bone (blue staining), and osteoid (orange staining) are shown. In the fourth week, the defect site comprised newly formed woven bone and osteoid in the I-nHAC/PLGA group. In contrast, small amounts of bone tissue around the scaffolds were observed in the other groups. In the eighth week, the woven bone was gradually transformed into lamellar bone with abundant mineralized area. A large number of mature lamellar bones were mineralized in the I-nHAC/PLGA group. In contrast, a similar regeneration process was observed in the other groups, but only thin trabecular bone formations were seen.

Based on Goldner's trichrome staining, significant differences were illustrated in bone formation using histomorphometric measurements. In the eighth week, the amounts of bone formed (BV/TV) in the nHAC, I-nHAC, nHAC/PLGA, and I-nHAC/PLGA groups were $31.3 \% \pm 3.2 \%, 32.7 \% \pm 4.3 \%$, $30.3 \% \pm 2.6 \%$, and $46.6 \% \pm 4.4 \%$, respectively (Figure 7 ). The amount of bone formation in the I-nHAC/PLGA group was significantly higher than those of the other groups. The amounts of bone formed were not significantly different among the nHAC, I-nHAC, and nHAC/PLGA groups.

\section{Discussion}

Despite a growing interest in the development of composite scaffolds for drug delivery, there is a need for a carrier that
nHAC

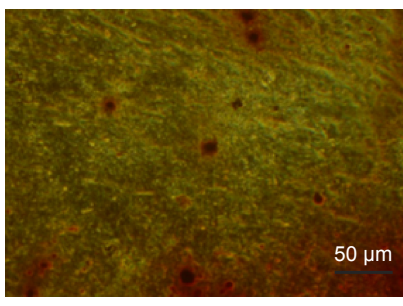

nHAC/PLGA

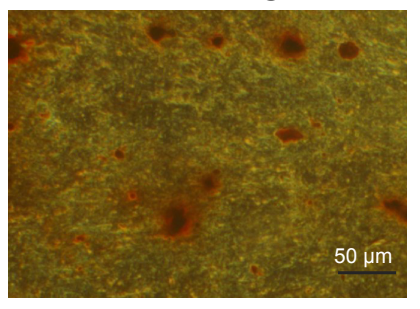

I-nHAC

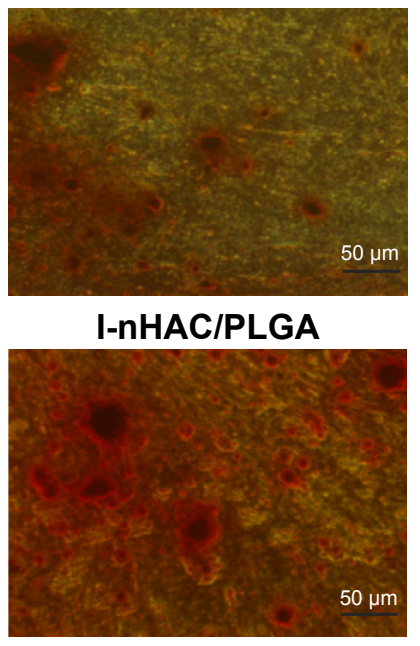

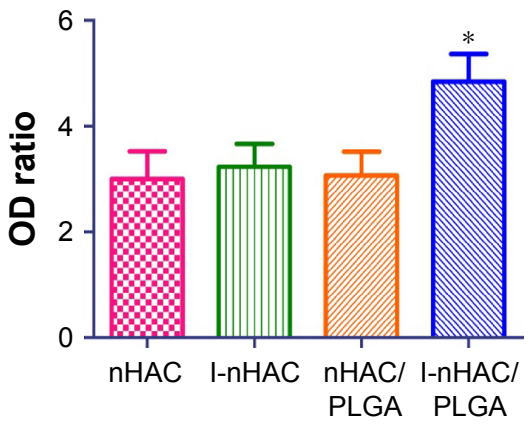

Figure 4 Mineralized formation in vitro examined by Alizarin Red staining. *Compared to nHAC. Differences are significant at $p<0.05$. Abbreviations: nHAC, nano-hydroxyapatite/collagen; OD, optical density; PLGA, poly lactic-co-glycolic-acid. 

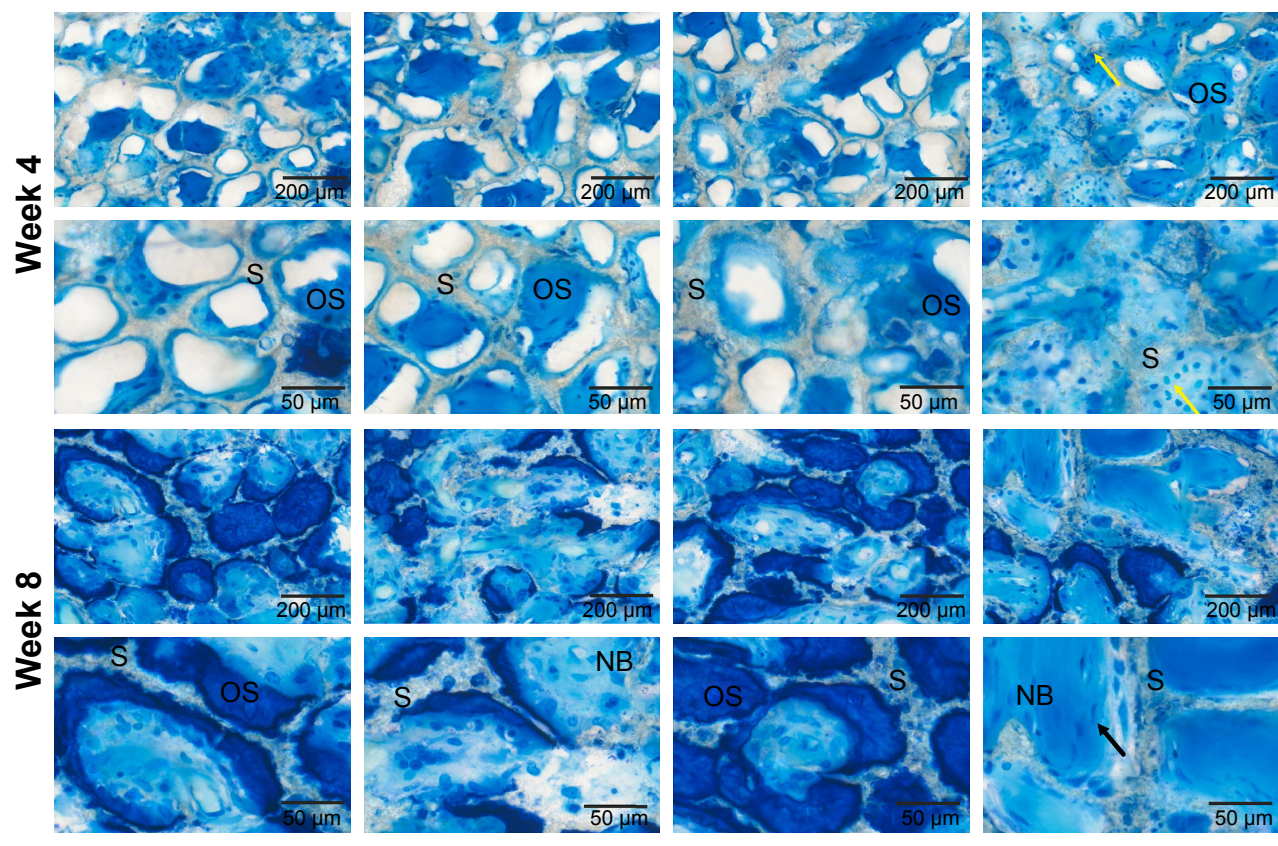

nHAC/PLGA

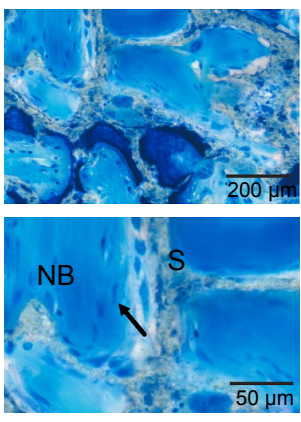

Figure 5 Histological evaluation of bone formation in mandible bone defects at the fourth and eighth week by toluidine blue staining (black arrows: osteocytes in bone lacuna, yellow arrows: osteoblasts).

Abbreviations: NB, new bone; nHAC, nano-hydroxyapatite/collagen; OS, osteoid; PLGA, poly lactic-co-glycolic-acid; S, composite scaffold.

is robust enough to maintain a three-dimensional space for new bone formation and provide a controlled release of bioactive factors. ${ }^{23}$

In this study, we incorporated uniform-sized, insulinloaded PLGA nanospheres into nHAC scaffolds to control the release of insulin. The physical characterizations of the composite scaffolds demonstrated that incorporation of nanospheres in nHAC scaffolds using this method did not significantly change the porosity, pore diameters, and compressive strengths of nHAC. The insulin release profile
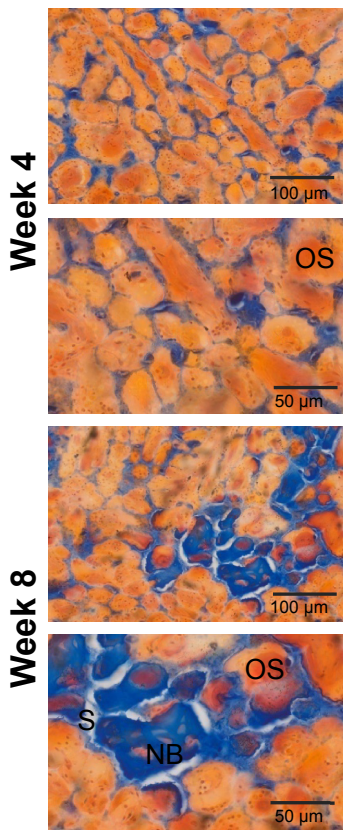

nHAC
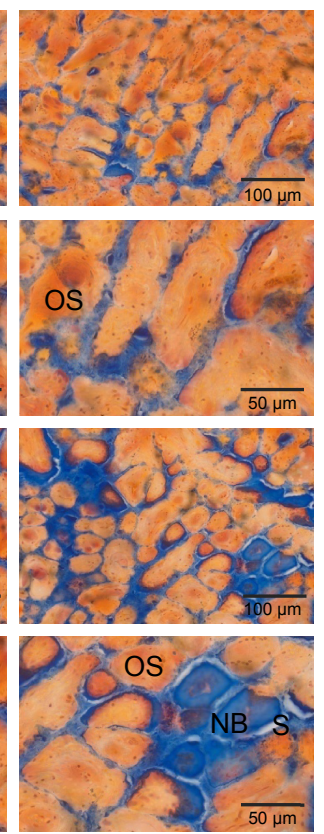

I-nHAC
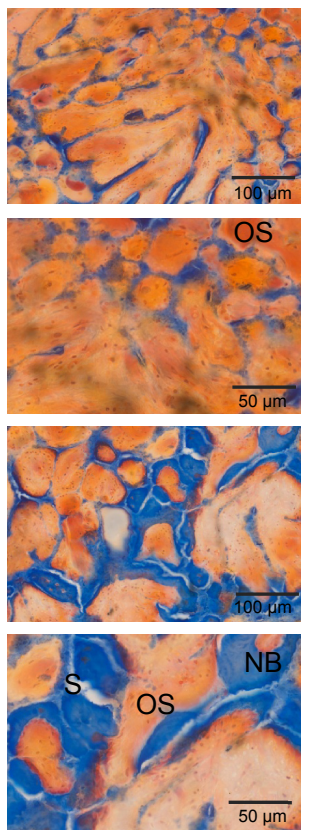

nHAC/PLGA
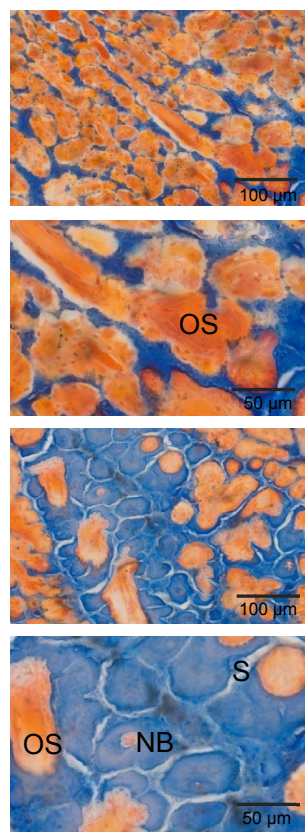

I-nHAC/PLGA

Figure 6 Histological evaluation of bone formation in mandible bone defects at the fourth and eighth week by Goldner's trichrome staining Abbreviations: NB, mineralized bone; nHAC, nano-hydroxyapatite/collagen; OS, osteoid; PLGA, poly lactic-co-glycolic-acid; S, composite scaffold. 


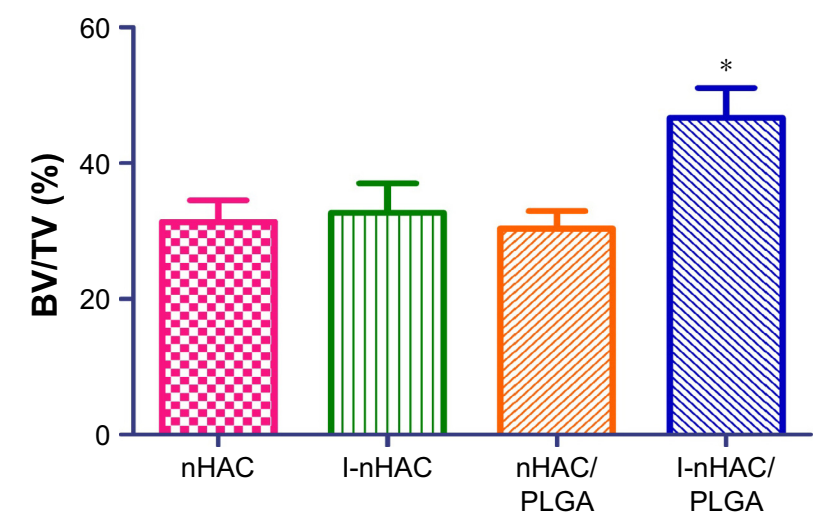

Figure 7 Histomorphometric measurements based on Goldner's trichrome staining at the eighth week postimplantation $(n=6$, mean $\pm S D)$. *Compared to $n H A C$. Differences are significant at $p<0.05$.

Abbreviations: BV/TV, bone volume per total volume; $\mathrm{nHAC}$, nano-hydroxyapatite/ collagen; PLGA, poly lactic-co-glycolic-acid.

from the composite scaffold significantly increased BMSC proliferation and ALP and OCN expression. The results indicated that this composite scaffold promoted active osteogenic status in BMSCs. Moreover, the bone regenerative capability of this composite scaffold was confirmed in a rabbit mandible critical size defect model. These results demonstrated that the combination of hydroxyapatite with a drug-loaded biodegradable polymer in an HAC/PLGA composite scaffold possessed the required structural stability and insulin delivery function.

Previous studies have suggested that the effective concentration of insulin for osteogenic differentiation of MSCs is in range of $1-1,000 \mathrm{nM} \cdot{ }^{24,25}$ However, the plasma half-life of insulin is only $5-15$ minutes; so, in vivo applications are limited due to short periods of insulin activity. ${ }^{26,27}$ In this study, we optimized controlled release of bioactive insulin from nanospheres within the composite scaffold and maintained high insulin concentration at the defect site. To investigate the influence of the composite scaffolds on cell proliferation and osteogenic differentiation, human BMSCs were cultured on the scaffolds. The results indicated that the number of cells and ALP concentration were significantly increased in the I-nHAC/PLGA group, which was consistent with previously studies. Thus, insulin could stimulate the proliferation of BMSCs in vitro. ${ }^{28} \mathrm{After} 21$ days of culture, calcium accumulation in the I-nHAC/PLGA group was significantly higher than in the other groups. This was because, during the primary bone formation stage, insulin released from the I-nHAC/PLGA scaffolds significantly stimulated osteogenesis and mineralization. These results were consistent with those of Fulzele and Clemens, who proved that insulin increased osteogenic differentiation by suppressing the Runx2 inhibitor Twist $2 .{ }^{4}$ Furthermore, in vivo results indicated that the insulin-loaded nHAC/PLGA scaffold significantly enhanced bone healing after 4 and 8 weeks postimplantation compared with other scaffolds. These data demonstrated that this insulin-loaded composite scaffold could substantially improve bone repair in rabbit critical size mandible defects.

In this study, uniform-sized, insulin-loaded PLGA nanospheres were loaded into a porous nHAC scaffold via a vacuum suction method. Many studies have attempted to prepare a scaffold with osteogenic inducer and hydroxyapatite. A variety of HAC coating techniques have been investigated, including electrophoretic deposition, electrodeposition, and plasma spray. A composite scaffold conjunct HAC and biocompatible polymers may provide an alternative method, which would also retain the bioactivity of HAC to promote bone growth. Son et al coated polyethyleneimine (PEI) on PLGA nanospheres surfaces. The PEI-coated PLGA nanospheres were immobilized on hydroxyapatite surfaces via electrostatic interactions. ${ }^{29}$ However, PEI has not been approved for clinical use due to its cytotoxicity. ${ }^{30}$ Compared with previous methods, the vacuum suction method described herein offers several advantages. This physical method does not affect insulin activity or have any cytotoxic effects during degradation.

Insulin has been widely used in clinics, so we believe that this scaffold has many potential advantages for patients. Moreover, the results of this study may not be limited to insulin. Bone morphogenetic proteins (BMPs) have been demonstrated to elicit new bone formation at both orthotopic and ectopic sites in animal models. However, BMPs are expensive and labile proteins. A direct injection of BMPs may diffuse more rapidly to the surrounding medium leading to burst delivery. ${ }^{31}$ In this study, I-nHAC/PLGA scaffolds characterized by controlled release of insulin were successfully prepared. These results also suggested that nHAC/ PLGA may be a promising carrier for BMP-2 and other growth factors.

\section{Conclusion}

A functional, insulin-loaded nHAC/PLGA composite scaffold was successfully developed to serve as an insulin delivery system and a three-dimensional scaffold to maintain cellular activities in the repair of bone defects. Bioactive insulin was successfully released from nanospheres within the scaffold, and the release kinetics of insulin was efficiently controlled. The composite scaffolds were shown to have favorable mechanical and structural properties for BMSC 
adherence, proliferation, and differentiation into osteoblasts. Implantation of the composite scaffolds into rabbit critical size defect models provided better tissue compatibility and higher bone restoration capacity, compared with defects that were either unfilled or filled with nHAC scaffolds alone.

\section{Acknowledgments}

This work was supported by grants from Shanxi Applied Basic Research Program Science - Youth Technology Research Fund (201701D221160), the China Postdoctoral Science Foundation, and the Startup Foundation for Doctors of Shanxi Medical University (BS03201639).

\section{Disclosure}

The authors report no conflicts of interest in this work.

\section{References}

1. Teotia AK, Gupta A, Raina DB, Lidgren L, Kumar A. Gelatinmodified bone substitute with bioactive molecules enhance cellular interactions and bone regeneration. ACS Appl Mater Interfaces. 2016; 8(17):10775-10787.

2. Gu W, Wu C, Chen J, Xiao Y. Nanotechnology in the targeted drug delivery for bone diseases and bone regeneration. Int J Nanomedicine. 2013;8(1):2305-2317.

3. Contaldo C, Myers TJ, Zucchini C, et al. Expression levels of insulin receptor substrate-1 modulate the osteoblastic differentiation of mesenchymal stem cells and osteosarcoma cells. Growth Factors. 2014; 32(1):41-52.

4. Fulzele K, Clemens TL. Novel functions for insulin in bone. Bone. 2012;50(2):452-456.

5. Kream BE, Smith MD, Canalis E, Raisz LG. Characterization of the effect of insulin on collagen synthesis in fetal rat bone. Endocrinology. 1985;116(1):296-302.

6. Pramojanee SN, Phimphilai M, Chattipakorn N, Chattipakorn SC. Possible roles of insulin signaling in osteoblasts. Endocr Res. 2014; 39(4):144-151.

7. Wang B, Song Y, Wang F, et al. Effects of local infiltration of insulin around titanium implants in diabetic rats. Br J Oral Maxillofac Surg. 2011;49(3):225-229.

8. Lock J, Nguyen TY, Liu H. Nanophase hydroxyapatite and poly(lactideco-glycolide) composites promote human mesenchymal stem cell adhesion and osteogenic differentiation in vitro. J Mater Sci Mater Med. 2012;23(10):2543-2552.

9. Guo X, Gough JE, Xiao P, Liu J, Shen Z. Fabrication of nanostructured hydroxyapatite and analysis of human osteoblastic cellular response. J Biomed Mater Res A. 2007;82(4):1022-1032.

10. Liao S, Tamura K, Zhu Y, et al. Human neutrophils reaction to the biodegraded nano-hydroxyapatite/collagen and nano-hydroxyapatite/ collagen/poly(L-lactic acid) composites. J Biomed Mater Res A. 2006; 76(5):820-825.

11. Ling LE, Feng L, Liu HC, et al. The effect of calcium phosphate composite scaffolds on the osteogenic differentiation of rabbit dental pulp stem cells. J Biomed Mater Res A. 2015;103(5):1732-1745.

12. Liu HC, E LL, Wang DS, et al. Reconstruction of alveolar bone defects using bone morphogenetic protein 2 mediated rabbit dental pulp stem cells seeded on nano-hydroxyapatite/collagen/poly(L-lactide). Tissue Eng Part A. 2011;17(19-20):2417-2433.
13. Qi F, Wu J, Yang T, Ma G, Su Z. Mechanistic studies for monodisperse exenatide-loaded PLGA microspheres prepared by different methods based on SPG membrane emulsification. Acta Biomater. 2014;10(10): $4247-4256$.

14. Wang X, Wu X, Xing H, et al. Porous nanohydroxyapatite/collagen scaffolds loading insulin PLGA particles for restoration of critical size bone defect. ACS Appl Mater Interfaces. 2017;9(13):11380-11391.

15. Quinlan E, López-Noriega A, Thompson E, Kelly HM, Cryan SA, O'Brien FJ. Development of collagen-hydroxyapatite scaffolds incorporating PLGA and alginate microparticles for the controlled delivery of rhBMP-2 for bone tissue engineering. $J$ Control Release. 2015;198:71-79.

16. Liao SS, Cui FZ, Zhang W, Feng QL. Hierarchically biomimetic bone scaffold materials: nano-HA/collagen/PLA composite. J Biomed Mater Res B. 2004;69(2):158-165.

17. Wang X, Zou X, Zhao J, et al. Site-specific characteristics of bone marrow mesenchymal stromal cells modify the effect of aging on the skeleton. Rejuvenation Res. 2016;19(5):351-361.

18. Zhang SM, Cui FZ, Liao SS, Zhu Y, Han L. Synthesis and biocompatibility of porous nano-hydroxyapatite/collagen/alginate composite. J Mater Sci Mater Med. 2003;14(7):641-645.

19. Tang W, Lin D, Yu Y, et al. Bioinspired trimodal macro/micro/nanoporous scaffolds loading rhBMP-2 for complete regeneration of critical size bone defect. Acta Biomater. 2016;32:309-323.

20. Wang X, Xing H, Zhang G, et al. Restoration of a critical mandibular bone defect using human alveolar bone-derived stem cells and porous nano-HA/collagen/PLA scaffold. Stem Cells Int. 2016; 2016:8741641.

21. Gundersen HJ, Bendtsen TF, Korbo L, et al. Some new, simple and efficient stereological methods and their use in pathological research and diagnosis. APMIS. 1988;96(5):379-394.

22. Stanford CM, Jacobson PA, Eanes ED, Lembke LA, Midura RJ. Rapidly forming apatitic mineral in an osteoblastic cell line (UMR 106-01 BSP). J Biol Chem. 1995;270(16):9420-9428.

23. Anderson JM, Patterson JL, Vines JB, Javed A, Gilbert SR, Jun HW. Biphasic peptide amphiphile nanomatrix embedded with hydroxyapatite nanoparticles for stimulated osteoinductive response. ACS Nano. 2011;5(12):9463-9479.

24. Wang L, Wang H, Xu X, Wang D, Liu H. Insulin facilitates osteoblast differentiation. Cell Biol Int. 2013;37(11):1157-1161.

25. Mueller MB, Blunk T, Appel B, et al. Insulin is essential for in vitro chondrogenesis of mesenchymal progenitor cells and influences chondrogenesis in a dose-dependent manner. Int Orthop. 2013;37(1): 153-158.

26. Malekzadeh B, Tengvall P, Ohrnell LO, Wennerberg A, Westerlund A. Effects of locally administered insulin on bone formation in nondiabetic rats. J Biomed Mater Res A. 2013;101(1):132-137.

27. Dedania J, Borzio R, Paglia D, et al. Role of local insulin augmentation upon allograft incorporation in a rat femoral defect model. J Orthop Res. 2011;29(1):92-99.

28. Pun KK, Lau P, Ho PW. The characterization, regulation, and function of insulin receptors on osteoblast-like clonal osteosarcoma cell line. J Bone Miner Res. 1989;4(6):853-862.

29. Son JS, Appleford M, Ong JL, et al. Porous hydroxyapatite scaffold with three-dimensional localized drug delivery system using biodegradable microspheres. J Control Release. 2011;153(2):133-140.

30. Wang S, Zhang J, Wang Y, Chen M. Hyaluronic acid-coated PEIPLGA nanoparticles mediated co-delivery of doxorubicin and miR542-3p for triple negative breast cancer therapy. Nanomedicine. 2016;12(2):411-420.

31. Niu X, Feng Q, Wang M, Guo X, Zheng Q. Porous nano-HA/collagen/ PLLA scaffold containing chitosan microspheres for controlled delivery of synthetic peptide derived from BMP-2. J Control Release. 2009;134(2):111-117. 
International Journal of Nanomedicine

Dovepress

\section{Publish your work in this journal}

The International Journal of Nanomedicine is an international, peerreviewed journal focusing on the application of nanotechnology in diagnostics, therapeutics, and drug delivery systems throughout the biomedical field. This journal is indexed on PubMed Central, MedLine, CAS, SciSearch ${ }^{\circledR}$, Current Contents ${ }^{\circledR} /$ Clinical Medicine,
Journal Citation Reports/Science Edition, EMBase, Scopus and the Elsevier Bibliographic databases. The manuscript management system is completely online and includes a very quick and fair peer-review system, which is all easy to use. Visit http://www.dovepress.com/ testimonials.php to read real quotes from published authors.

Submit your manuscript here: http://www.dovepress.com/international-journal-of-nanomedicine-journal 